Citation: Pandir, Y., Ulusoy, H. " The Multi-Wave Method For Exact Solutions of Nonlinear Partial Differential Equations". Journal of Engineering Technology and Applied Sciences 2 (1) 2017 : 13-26

\title{
THE MULTI-WAVE METHOD FOR EXACT SOLUTIONS OF NONLINEAR PARTIAL DIFFERENTIAL EQUATIONS
}

\author{
Yusuf Pandir ${ }^{a^{*}}$, Halime Ulusoy ${ }^{b}$ \\ $a^{*}$ Department of Mathematics, Faculty of Science and Arts, Bozok University, Yozgat Turkey \\ yusuf.pandir@bozok.edu.tr (corresponding author) \\ ${ }^{b}$ Graduate School of Natural and Applied Sciences, Bozok University, Yozgat, Turkey \\ ulusoyhalime@gmail.com
}

\begin{abstract}
In this research, we use the multi-wave method to obtain new exact solutions for generalized forms of the $5^{\text {th }}$ order $\mathrm{KdV}$ equation and the fifth order $\mathrm{KdV}(\mathrm{fKdV})$ equation with power law nonlinearity. Computations are performed with the help of the mathematics software Mathematica. Then periodic wave solutions, bright soliton solutions and rational function solutions with free parameters are obtained by this approach. It is shown that this method is very useful and effective.
\end{abstract}

Keywords: Multi-wave method, KdV equation, Soliton solutions.

\section{Introduction}

The research of exact solutions to nonlinear evolution equations is very important, because these problems appear in fluid mechanics, plasma physics, optical fibers, biology, solid state physics, chemical kinematics, chemical physics, and so on. For this reason, a lot of mathematical methods have been developed in this area. In order to find the wave solutions, many methods were attempted in literature such as solitary wave ansatz method [1], Hirota's bilinear transformation method [2,3], exp-function method [4,5], $\left(G^{\prime} / G\right)$-expansion method [6], trial equation method [7], and so on. Also, Dai et al. [8] proposed extended three-soliton method. Then, Shi et al. [9-11] constructed a novel approach, namely, multi-wave method to find new exact solutions for nonlinear problems and extend Dai et al.'s work [8].

In this work, we apply the multi-wave method in order to find new results to nonlinear problems. We demonstrate an application of multi-wave method for finding exact solutions of two generalized nonlinear evolution equations. One of these equations is $5^{\text {th }}$ order $\mathrm{KdV}$ equation [12]

$$
q_{t}+a q^{m} q_{x}+b q^{2 m} q_{x}+c q_{x x x}+k q_{x x x x x}=0,
$$


where $a, b, c$ and $k$ are all constants. The first term is the evolution term, while the second and third terms together form the nonlinear terms. Finally, the coefficients of $c$ and $k$ are the third and fifth order dispersion terms, respectively. The other one is the dimensionless form of the fifth order $\mathrm{KdV}(\mathrm{fKdV})$ equation with power law nonlinearity [13]

$$
q_{t}+a q_{x x x}+b q_{x x x x x}-c\left(q^{n+1}\right)_{x}=0
$$

where $a, b$ and $c$ are constants parameters and $n>0$.

\section{The bilinear form of 5 th order KdV equation}

Let's suppose $m=1$ in Eq. (1) and

$$
w=(\ln f)_{x}, \quad q=w_{x}=(\ln f)_{x x}=0 .
$$

Substituting $q=w_{x}$ into Eq. (1), we can get

$$
w_{x t}+a w_{x} w_{x x}+b w_{x}^{2} w_{x x}+c w_{x x x x}+k w_{x x x x x x}=0 .
$$

Integrating Eq. (4) with respect to $x$ and equating the integral constant to zero, we have

$$
w_{t}+\frac{a}{2} w_{x}^{2}+b w_{x}^{3}+c w_{x x x}+k w_{x x x x x}=0
$$

Substituting Eq. (3) into Eq. (5), we can reduce Eq. (1) into the bilinear forms as follows

$$
\begin{aligned}
& \left(D_{t} D_{x}+c D_{x}^{4}-\frac{b}{360} D_{x}^{6}\right)(f \cdot f)=0, \\
& \left(\frac{a}{2} D_{x}^{2}-3 c D_{x}^{2}+\frac{b}{24} D_{x}^{4}\right)(f \cdot f)=0,
\end{aligned}
$$

where $f=f(x, t)$ is an unknown real function, the bilinear operator $D_{x}^{m} D_{t}^{n}$ is defined as

$$
D_{x}^{m} D_{t}^{n} a b=\left.\left(\frac{\partial}{\partial x}-\frac{\partial}{\partial x^{\prime}}\right)^{m}\left(\frac{\partial}{\partial t}-\frac{\partial}{\partial t^{\prime}}\right)^{n} a(x, t) b\left(x^{\prime}, t^{\prime}\right)\right|_{x=x^{\prime} t=t^{\prime}}=0 .
$$

\section{An application of the multi-wave method for 5th order KdV equation}

We first consider the test function of extended four-soliton method as follows:

$$
f(x, t)=a_{1} \cos \left(\xi_{1}\right)+a_{2} \sin \left(\xi_{2}\right)+a_{3} \cosh \left(\xi_{3}\right)+\exp \left(-\xi_{4}\right)+a_{4} \exp \left(\xi_{4}\right),
$$

where $\xi_{i}=p_{i} x+r_{i} t, i=1,2,3,4$. Substituting (8) into (3), we can write the form of general solution of Eq. (1)

$$
\begin{aligned}
& q=\frac{-a_{1} p_{1}^{2} \cos \left(p_{1} x+r_{1} t\right)-a_{2} p_{2}^{2} \sin \left(p_{2} x+r_{2} t\right)+a_{3} p_{3}^{2} \cosh \left(p_{3} x+r_{3} t\right)+p_{4}^{2} \exp \left(-p_{4} x-r_{4} t\right)+a_{4} p_{4}^{2} \exp \left(p_{4} x+r_{4} t\right)}{a_{1} \cos \left(p_{1} x+r_{1} t\right)+a_{2} \sin \left(p_{2} x+r_{2} t\right)+a_{3} \cosh \left(p_{3} x+r_{3} t\right)+\exp \left(-p_{4} x-r_{4} t\right)+a_{4} \exp \left(p_{4} x+r_{4} t\right)}- \\
& \left(\frac{-a_{1} p_{1} \sin \left(p_{1} x+r_{1} t\right)+a_{2} p_{2} \cos \left(p_{2} x+r_{2} t\right)+a_{3} p_{3} \sinh \left(p_{3} x+r_{3} t\right)-p_{4} \exp \left(-p_{4} x-r_{4} t\right)+a_{4} p_{4} \exp \left(p_{4} x+r_{4} t\right)}{a_{1} \cos \left(p_{1} x+r_{1} t\right)+a_{2} \sin \left(p_{2} x+r_{2} t\right)+a_{3} \cosh \left(p_{3} x+r_{3} t\right)+\exp \left(-p_{4} x-r_{4} t\right)+a_{4} \exp \left(p_{4} x+r_{4} t\right)}\right)^{2}
\end{aligned}
$$


Using Eqs. (9) and (6), we obtain a set of algebraic equations for $a_{1}, a_{2}, a_{3}, a_{4}, p_{1}, p_{2}, p_{3}, p_{4}, r_{1}, r_{2}, r_{3}, r_{4}$. Solving these systems with the aid of Mathematica, we obtain the following solutions, respectively:

\section{Case 1.}

$a_{4}=p_{1}=p_{2}=p_{3}=r_{1}=r_{2}=r_{3}=0, p_{4}=2 \sqrt{\frac{18 c-3 a}{b}}, r_{4}=-4 \sqrt{3}(a+24 c)\left(\frac{6 c-a}{5 b}\right)^{\frac{3}{2}}$,

where $a_{1}, a_{3}$ are free parameters. Substituting (10) into Eq. (9) yields the following wave solution of Eq. (1)

$$
q(x, t)=\frac{(72 c-12 a)\left(a_{1}+a_{3}\right) \exp \left(-\xi_{4}\right)}{b\left(a_{1}+a_{3}+\exp \left(\xi_{4}\right)\right)}
$$

where $\xi_{4}=2 \sqrt{\frac{18 c-3 a}{b}} x-4 \sqrt{3}(a+24 c)\left(\frac{6 c-a}{5 b}\right)^{\frac{3}{2}} t$

\section{Case 2.}

$$
\begin{gathered}
p_{1}=p_{2}=\sqrt{\frac{3 a-18 c}{b}}, \quad p_{3}=p_{4}=\sqrt{\frac{18 c-3 a}{b}}, \quad r_{1}=r_{2}=\sqrt{3}(a+54 c)\left(\frac{a-6 c}{5 b}\right)^{\frac{3}{2}}, \\
r_{3}=r_{4}=-\sqrt{3}(a+54 c)\left(\frac{6 c-a}{5 b}\right)^{\frac{3}{2}},
\end{gathered}
$$

where $a_{1}, p_{2}$ and $r_{2}$ are free parameters. Substituting (12) into Eq. (9) yields the exact solution

$q(x, t)=\frac{18 c-3 a}{b}+\left(\frac{a_{1} \sqrt{\frac{3 a-18 c}{b}} \sin \left(\xi_{1}\right)-a_{2} \sqrt{\frac{3 a-18 c}{b}} \cos \left(\xi_{2}\right)-a_{3} \sqrt{\frac{18 c-3 a}{b}} \sinh \left(\xi_{3}\right)+\sqrt{\frac{18 c-3 a}{b}} \exp \left(-\xi_{4}\right)-a_{4} \sqrt{\frac{18 c-3 a}{b}} \exp \left(\xi_{4}\right)}{a_{1} \cos \left(\xi_{1}\right)+a_{2} \sin \left(\xi_{2}\right)+a_{3} \cosh \left(\xi_{3}\right)+\exp \left(-\xi_{4}\right)+a_{4} \exp \left(\xi_{4}\right)}\right)^{2}$

where $\xi_{1}=\xi_{2}=\sqrt{\frac{3 a-18 c}{b}} x+\sqrt{3}(a+54 c)\left(\frac{a-6 c}{5 b}\right)^{\frac{3}{2}} t, \xi_{3}=\xi_{4}=\sqrt{\frac{18 c-3 a}{b}} x-\sqrt{3}(a+54 c)\left(\frac{6 c-a}{5 b}\right)^{\frac{3}{2}} t$.

Also, if we take $\frac{b}{3}= \pm(a-6 c)$ and $a_{3}=-1, a_{4}=1$, then Eq. (13) can be reduced to the following form:

$$
q(x, t)=1-\left(\frac{-a_{1} \sin \left(\xi_{1}\right)+a_{2} \cos \left(\xi_{2}\right)+\sinh \left(\xi_{4}\right)}{a_{1} \cos \left(\xi_{1}\right)+a_{2} \sin \left(\xi_{2}\right)+\cosh \left(\xi_{4}\right)}\right)^{2} .
$$

\section{Case 3.}

$a_{1}=a_{4}=0, p_{2}=p_{3}=0, r_{2}=r_{3}=0, p_{4}=\sqrt{\frac{72 c-12 a}{b}}, r_{4}=-2 \sqrt{3}(a+54 c)\left(\frac{6 c-a}{5 b}\right)^{\frac{3}{2}}$,

where $a_{3}$ is a free parameter. Substituting (15) into Eq. (9), we can obtain the following exact solitary solution:

$$
q(x, t)=\frac{(72 c-12 a) a_{3} \exp \left(-\xi_{4}\right)}{b\left(a_{3}+\exp \left(\xi_{4}\right)\right)^{2}}
$$


where $\xi_{4}=\sqrt{\frac{72 c-12 a}{b}} x-2 \sqrt{3}(a+54 c)\left(\frac{6 c-a}{5 b}\right)^{\frac{3}{2}} t$

\section{Case 4.}

$$
\begin{gathered}
a_{1}=0, p_{2}=\sqrt{\frac{3 a-18 c}{b}}, \quad p_{3}=p_{4}=\sqrt{\frac{18 c-3 a}{b}}, \quad r_{2}=\sqrt{3}(a+54 c)\left(\frac{a-6 c}{5 b}\right)^{\frac{3}{2}}, \\
r_{3}=r_{4}=-\sqrt{3}(a+54 c)\left(\frac{6 c-a}{5 b}\right)^{\frac{3}{2}},
\end{gathered}
$$

where $a_{2}, a_{3}$ and $a_{4}$ are free parameters. Substituting (17) into Eq. (9) yields the exact solution

$q(x, t)=\frac{18 c-3 a}{b}-\left(\frac{a_{2} \sqrt{\frac{3 a-18 c}{b}} \cos \left(\xi_{2}\right)+a_{3} \sqrt{\frac{18 c-3 a}{b}} \sinh \left(\xi_{3}\right)-\sqrt{\frac{18 c-3 a}{b}} \exp \left(-\xi_{4}\right)+a_{4} \sqrt{\frac{18 c-3 a}{b}} \exp \left(\xi_{4}\right)}{a_{2} \sin \left(\xi_{2}\right)+a_{3} \cosh \left(\xi_{3}\right)+\exp \left(-\xi_{4}\right)+a_{4} \exp \left(\xi_{4}\right)}\right)^{2}$

where $\xi_{2}=\sqrt{\frac{3 a-18 c}{b}} x+\sqrt{3}(a+54 c)\left(\frac{a-6 c}{5 b}\right)^{\frac{3}{2}} t, \xi_{3}=\xi_{4}=\sqrt{\frac{18 c-3 a}{b}} x-\sqrt{3}(a+54 c)\left(\frac{6 c-a}{5 b}\right)^{\frac{3}{2}} t$.

Also, if we take $\frac{b}{3}= \pm(6 c-a)$ and $a_{3}=-1, a_{4}=1$, then Eq. (18) can be reduced to the following form:

$$
q(x, t)=1-\left(\frac{a_{2} \cos \left(\xi_{2}\right)+\sinh \left(\xi_{4}\right)}{a_{2} \sin \left(\xi_{2}\right)+\cosh \left(\xi_{4}\right)}\right)^{2} .
$$

\section{Case 5.}

$$
\begin{gathered}
a_{2}=0, p_{1}=\sqrt{\frac{3 a-18 c}{b}}, \quad p_{3}=p_{4}=\sqrt{\frac{18 c-3 a}{b}}, \quad r_{1}=\sqrt{3}(a+54 c)\left(\frac{a-6 c}{5 b}\right)^{\frac{3}{2}}, \\
r_{3}=r_{4}=-\sqrt{3}(a+54 c)\left(\frac{6 c-a}{5 b}\right)^{\frac{3}{2}},
\end{gathered}
$$

where $a_{1}, a_{3}$ and $a_{4}$ are free parameters. Substituting (20) into Eq. (9), we can write the exact solution

$$
q(x, t)=\frac{18 c-3 a}{b}-\left(\frac{-a_{1} \sqrt{\frac{3 a-18 c}{b}} \sin \left(\xi_{1}\right)+a_{3} \sqrt{\frac{18 c-3 a}{b}} \sinh \left(\xi_{3}\right)-\sqrt{\frac{18 c-3 a}{b}} \exp \left(-\xi_{4}\right)+a_{4} \sqrt{\frac{18 c-3 a}{b}} \exp \left(\xi_{4}\right)}{a_{1} \cos \left(\xi_{1}\right)+a_{3} \cosh \left(\xi_{3}\right)+\exp \left(-\xi_{4}\right)+a_{4} \exp \left(\xi_{4}\right)}\right)^{2}
$$

where $\xi_{1}=\sqrt{\frac{3 a-18 c}{b}} x+\sqrt{3}(a+54 c)\left(\frac{a-6 c}{5 b}\right)^{\frac{3}{2}} t, \xi_{3}=\xi_{4}=\sqrt{\frac{18 c-3 a}{b}} x-\sqrt{3}(a+54 c)\left(\frac{6 c-a}{5 b}\right)^{\frac{3}{2}} t$.

Also, if we take $\frac{b}{3}= \pm(6 c-a)$ and $a_{3}=-1, a_{4}=1$, then Eq. (21) can be reduced to the following form:

$$
q(x, t)=1-\left(\frac{-a_{1} \sin \left(\xi_{1}\right)+\sinh \left(\xi_{4}\right)}{a_{1} \cos \left(\xi_{1}\right)+\cosh \left(\xi_{4}\right)}\right)^{2} .
$$




\section{Case 6.}

$$
\begin{gathered}
a_{3}=0, p_{1}=p_{2}=\sqrt{\frac{3 a-18 c}{b}}, \quad p_{3}=p_{4}=\sqrt{\frac{18 c-3 a}{b}}, \quad r_{1}=r_{1}=\sqrt{3}(a+54 c)\left(\frac{a-6 c}{5 b}\right)^{\frac{3}{2}}, \\
r_{3}=r_{4}=-\sqrt{3}(a+54 c)\left(\frac{6 c-a}{5 b}\right)^{\frac{3}{2}}
\end{gathered}
$$

where $a_{1}, a_{2}$ and $a_{4}$ are free parameters. Substituting (23) into Eq. (9) yields the solitary solution

$$
q(x, t)=\frac{18 c-3 a}{b}-\left(\frac{-a_{1} \sqrt{\frac{3 a-18 c}{b}} \sin \left(\xi_{1}\right)+a_{2} \sqrt{\frac{3 a-18 c}{b}} \cos \left(\xi_{2}\right)-\sqrt{\frac{18 c-3 a}{b}} \exp \left(-\xi_{4}\right)+a_{4} \sqrt{\frac{18 c-3 a}{b}} \exp \left(\xi_{4}\right)}{a_{1} \cos \left(\xi_{1}\right)+a_{2} \sin \left(\xi_{2}\right)+\exp \left(-\xi_{4}\right)+a_{4} \exp \left(\xi_{4}\right)}\right)^{2}
$$

where $\xi_{1}=\xi_{2}=\sqrt{\frac{3 a-18 c}{b}} x+\sqrt{3}(a+54 c)\left(\frac{a-6 c}{5 b}\right)^{\frac{3}{2}} t, \xi_{4}=\sqrt{\frac{18 c-3 a}{b}} x-\sqrt{3}(a+54 c)\left(\frac{6 c-a}{5 b}\right)^{\frac{3}{2}} t$.

Also, if we take $\frac{b}{3}= \pm(6 c-a)$ and $a_{4}=1$, then Eq. (24) can be reduced to the following form:

$$
q(x, t)=1-\left(\frac{-a_{1} \sin \left(\xi_{1}\right)+a_{2} \cos \left(\xi_{2}\right)+2 \sinh \left(\xi_{4}\right)}{a_{1} \cos \left(\xi_{1}\right)+a_{2} \sin \left(\xi_{2}\right)+2 \cosh \left(\xi_{4}\right)}\right)^{2} .
$$

\section{Case 7.}

$$
a_{1}=a_{2}=0, \quad p_{3}=p_{4}=\sqrt{\frac{18 c-3 a}{b}}, r_{3}=r_{4}=-\sqrt{3}(a+54 c)\left(\frac{6 c-a}{5 b}\right)^{\frac{3}{2}},
$$

where $a_{3}, a_{4}$ are free parameters. Substituting (26) into Eq. (9) yields the following wave solution to Eq.(1)

$$
q(x, t)=\frac{18 c-3 a}{b}-\left(\frac{a_{3} \sqrt{\frac{18 c-3 a}{b}} \sinh \left(\xi_{3}\right)-\sqrt{\frac{18 c-3 a}{b}} \exp \left(-\xi_{4}\right)+a_{4} \sqrt{\frac{18 c-3 a}{b}} \exp \left(\xi_{4}\right)}{a_{3} \cosh \left(\xi_{3}\right)+\exp \left(-\xi_{4}\right)+a_{4} \exp \left(\xi_{4}\right)}\right)^{2}
$$

where $\xi_{3}=\xi_{4}=\sqrt{\frac{18 c-3 a}{b}} x-\sqrt{3}(a+54 c)\left(\frac{6 c-a}{5 b}\right)^{\frac{3}{2}} t$. Also, if we take $\frac{b}{3}= \pm(6 c-a)$ and $a_{3}=$ $-1, a_{4}=1$, then Eq. (27) can be reduced to the following form:

$$
q(x, t)=1-\tanh ^{2}\left(\xi_{3}\right)=\operatorname{sech}^{2}\left(\xi_{3}\right) .
$$

\section{Case 8.}

$$
\begin{gathered}
\boldsymbol{a}_{1}=\boldsymbol{a}_{3}=\mathbf{0}, \boldsymbol{p}_{2}=\sqrt{\frac{3 a-18 c}{b}}, \boldsymbol{p}_{4}=\sqrt{\frac{18 c-3 a}{b}} \\
r_{2}=\sqrt{3}(a+54 c)\left(\frac{a-6 c}{5 b}\right)^{\frac{3}{2}}, r_{4}=-\sqrt{3}(a+54 c)\left(\frac{6 c-a}{5 b}\right)^{\frac{3}{2}},
\end{gathered}
$$

where $a_{2}$ and $a_{4}$ are free parameters. Substituting (29) into Eq. (9), we have the following wave solution: 


$$
q(x, t)=\frac{18 c-3 a}{b}-\left(\frac{a_{2} \sqrt{\frac{3 a-18 c}{b}} \cos \left(\xi_{2}\right)-\sqrt{\frac{18 c-3 a}{b}} \exp \left(-\xi_{4}\right)+a_{4} \sqrt{\frac{18 c-3 a}{b}} \exp \left(\xi_{4}\right)}{a_{2} \sin \left(\xi_{2}\right)+\exp \left(-\xi_{4}\right)+a_{4} \exp \left(\xi_{4}\right)}\right)^{2}
$$

where $\xi_{2}=\sqrt{\frac{3 a-18 c}{b}} x-\sqrt{3}(a+54 c)\left(\frac{a-6 c}{5 b}\right)^{\frac{3}{2}} t, \xi_{4}=\sqrt{\frac{18 c-3 a}{b}} x-\sqrt{3}(a+54 c)\left(\frac{6 c-a}{5 b}\right)^{\frac{3}{2}} t$. Also, if we take $\frac{b}{3}= \pm(6 c-a)$ and $a_{4}=1$, then Eq. (30) can be reduced to the following form:

$$
q(x, t)=1-\left(\frac{a_{2} \cos \left(\xi_{2}\right)+2 \sinh \left(\xi_{4}\right)}{a_{2} \sin \left(\xi_{2}\right)+2 \cosh \left(\xi_{4}\right)}\right)^{2} .
$$

\section{Case 9.}

$$
\begin{gathered}
\boldsymbol{a}_{2}=\boldsymbol{a}_{3}=\mathbf{0}, \boldsymbol{p}_{1}=\sqrt{\frac{\mathbf{3 a - 1 8 c}}{b}}, \quad \boldsymbol{p}_{4}=\sqrt{\frac{\mathbf{1 8 c - 3 a}}{b}} \\
r_{1}=\sqrt{3}(a+54 c)\left(\frac{a-6 c}{5 b}\right)^{\frac{3}{2}}, r_{4}=-\sqrt{3}(a+54 c)\left(\frac{6 c-a}{5 b}\right)^{\frac{3}{2}}
\end{gathered}
$$

where $a_{1}$ and $a_{4}$ are free parameters. Substituting (32) into Eq. (9) yields the solitary solution

$$
q(x, t)=\frac{18 c-3 a}{b}-\left(\frac{-a_{1} \sqrt{\frac{3 a-18 c}{b}} \sin \left(\xi_{1}\right)-\sqrt{\frac{18 c-3 a}{b}} \exp \left(-\xi_{4}\right)+a_{4} \sqrt{\frac{18 c-3 a}{b}} \exp \left(\xi_{4}\right)}{a_{1} \cos \left(\xi_{1}\right)+\exp \left(-\xi_{4}\right)+a_{4} \exp \left(\xi_{4}\right)}\right)^{2},
$$

where $\xi_{1}=\sqrt{\frac{3 a-18 c}{b}} x-\sqrt{3}(a+54 c)\left(\frac{a-6 c}{5 b}\right)^{\frac{3}{2}} t, \xi_{4}=\sqrt{\frac{18 c-3 a}{b}} x-\sqrt{3}(a+54 c)\left(\frac{6 c-a}{5 b}\right)^{\frac{3}{2}} t$. Also, if we take $\frac{b}{3}= \pm(6 c-a)$ and $a_{4}=1$, then Eq. (33) can be reduced to the following form:

$$
q(x, t)=1-\left(\frac{-a_{1} \sin \left(\xi_{1}\right)+2 \sinh \left(\xi_{4}\right)}{a_{1} \cos \left(\xi_{1}\right)+2 \cosh \left(\xi_{4}\right)}\right)^{2}
$$

\section{Case 10.}

$$
a_{1}=a_{2}=a_{3}=0, \quad p_{4}=\sqrt{\frac{18 c-3 a}{b}}, \quad r_{4}=-\sqrt{3}(a+54 c)\left(\frac{6 c-a}{5 b}\right)^{\frac{3}{2}},
$$

where $a_{4}$ is a free parameter. Substituting (35) into Eq. (9), we can obtain soliton solution as follows:

$$
q(x, t)=\frac{18 c-3 a}{b}-\left(\frac{-\sqrt{\frac{18 c-3 a}{b}} \exp \left(-\xi_{4}\right)+a_{4} \sqrt{\frac{18 c-3 a}{b}} \exp \left(\xi_{4}\right)}{\exp \left(-\xi_{4}\right)+a_{4} \exp \left(\xi_{4}\right)}\right)^{2}
$$

where $\xi_{4}=\sqrt{\frac{18 c-3 a}{b}} x-\sqrt{3}(a+54 c)\left(\frac{6 c-a}{5 b}\right)^{\frac{3}{2}} t$. Also, if we take $\frac{b}{3}= \pm(6 c-a)$ and $a_{4}=1$, then Eq. (36) can be reduced to the following soliton solution

$$
q(x, t)=\operatorname{sech}^{2}\left(\xi_{4}\right)
$$



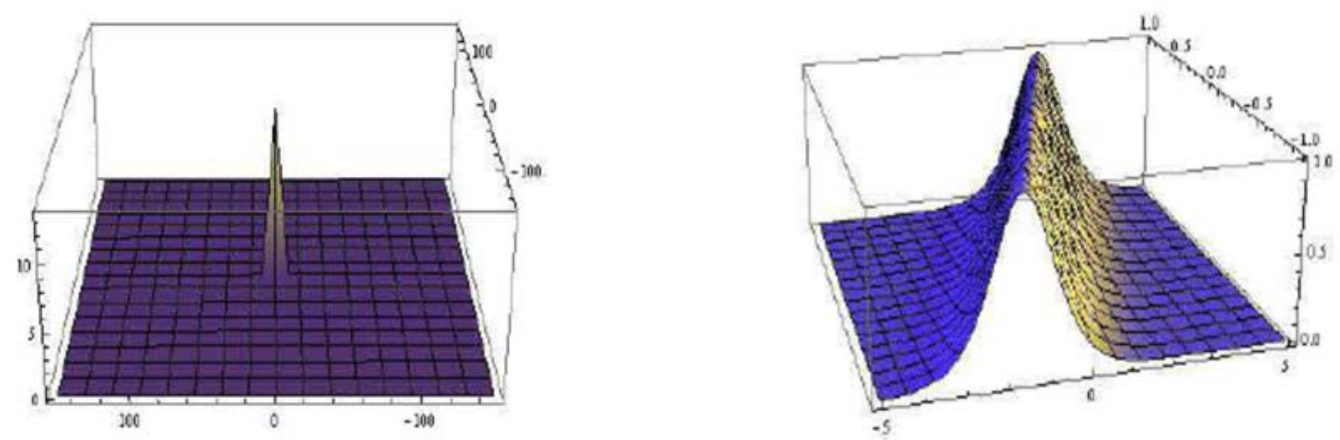

Figure 1. Solitary solution; Bright soliton

\section{The bilinear form of fKdV equation}

If $n=2$, then Eq. (2) can be converted into

$$
q_{t}+a q_{x x x}+b q_{x x x x x}-c\left(q^{3}\right)_{x}=0 .
$$

Suppose that

$$
w=(\ln f)_{x}, \quad q=w_{x}=(\ln f)_{x x}
$$

and substituting it into Eq. (38), we can get

$$
w_{x t}+a w_{x x x x}+b w_{x x x x x x}-c\left(w_{x}^{3}\right)_{x}=0 .
$$

Integrate $\mathrm{x}$ one time, and let the integral constant is zero, we have

$$
w_{t}+a w_{x x x}+b w_{x x x x x}-c w_{x}^{3}=0 .
$$

Substituting Eq. (39) into Eq. (41), we can reduce Eq. (38) into the bilinear forms as follows

$$
\begin{aligned}
& \left(D_{t} D_{x}+a D_{x}^{4}+\frac{c}{15} D_{x}^{6}\right)(f \cdot f)=0, \\
& D_{x}^{2}(f \cdot f) \cdot\left(-3 a D_{x}^{2}-c D_{x}^{4}\right)(f \cdot f)=0,
\end{aligned}
$$

where $f=f(x, t)$ is an unknown real function, $15 b=c$ and the bilinear operator $D_{x}^{m} D_{t}^{n}$ is defined as

$$
D_{x}^{m} D_{t}^{n} a b=\left.\left(\frac{\partial}{\partial x}-\frac{\partial}{\partial x^{\prime}}\right)^{m}\left(\frac{\partial}{\partial t}-\frac{\partial}{\partial t^{\prime}}\right)^{n} a(x, t) b\left(x^{\prime}, t^{\prime}\right)\right|_{x=x^{\prime} t=t^{\prime}}=0
$$

If we take

then Eq. (42) is converted into

$$
D_{x}^{4}(f \cdot f)=\frac{-3 a}{c} D_{x}^{2}(f \cdot f)
$$

$$
\left(D_{t} D_{x}-\frac{3 a}{c} D_{x}^{2}+\frac{c}{15} D_{x}^{6}\right)(f \cdot f)=0 .
$$




\section{The application of the multi-wave solutions method for solving the fKdV equation}

For this problem, we can use test function of extended four-soliton method

$$
f(x, t)=a_{1} \cos \left(\xi_{1}\right)+a_{2} \sin \left(\xi_{2}\right)+a_{3} \cosh \left(\xi_{3}\right)+\exp \left(-\xi_{4}\right)+a_{4} \exp \left(\xi_{4}\right),
$$

where $\xi_{i}=p_{i} x+r_{i} t, i=1,2,3,4$. Substituting (46) into (45), we can write the form of general solution of the $\mathrm{fKdV}$ equation

$$
\begin{aligned}
& q=\frac{-a_{1} p_{1}^{2} \cos \left(p_{1} x+r_{1} t\right)-a_{2} p_{2}^{2} \sin \left(p_{2} x+r_{2} t\right)+a_{3} p_{3}^{2} \cosh \left(p_{3} x+r_{3} t\right)+p_{4}^{2} \exp \left(-p_{4} x-r_{4} t\right)+a_{4} p_{4}^{2} \exp \left(p_{4} x+r_{4} t\right)}{a_{1} \cos \left(p_{1} x+r_{1} t\right)+a_{2} \sin \left(p_{2} x+r_{2} t\right)+a_{3} \cosh \left(p_{3} x+r_{3} t\right)+\exp \left(-p_{4} x-r_{4} t\right)+a_{4} \exp \left(p_{4} x+r_{4} t\right)}- \\
& \left(\frac{-a_{1} p_{1} \sin \left(p_{1} x+r_{1} t\right)+a_{2} p_{2} \cos \left(p_{2} x+r_{2} t\right)+a_{3} p_{3} \sinh \left(p_{3} x+r_{3} t\right)-p_{4} \exp \left(-p_{4} x-r_{4} t\right)+a_{4} p_{4} \exp \left(p_{4} x+r_{4} t\right)}{a_{1} \cos \left(p_{1} x+r_{1} t\right)+a_{2} \sin \left(p_{2} x+r_{2} t\right)+a_{3} \cosh \left(p_{3} x+r_{3} t\right)+\exp \left(-p_{4} x-r_{4} t\right)+a_{4} \exp \left(p_{4} x+r_{4} t\right)}\right)
\end{aligned}
$$

Substituting Eq. (47) into Eq. (45), and equating the coefficients of all powers of polynomials, we can find a set of algebraic equations for $a_{1}, a_{2}, a_{3}, a_{4}, p_{1}, p_{2}, p_{3}, p_{4}, r_{1}, r_{2}, r_{3}, r_{4}$. Solving these algebraic equations with the aid of Mathematica, we obtain the exact solutions as follows:

\section{Case 2.}

$$
a_{4}=0, p_{1}=p_{2}=p_{3}=r_{1}=r_{2}=r_{3}=0, r_{4}=p_{4}\left(\frac{3 a^{2}-b c p_{4}^{4}}{c}\right)
$$

where $a_{1}, a_{3}, p_{4}$ are free parameters. Substituting (48) into Eq. (47) yields the following wave solution

$$
q(x, t)=p_{4}^{2}\left(\frac{\left(a_{1}+a_{3}\right) \exp \left(-\xi_{4}\right)}{\left(a_{1}+a_{3}+\exp \left(\xi_{4}\right)\right)^{2}}\right)
$$

where $\xi_{4}=p_{4}\left(x-\frac{b c p_{4}^{4}-3 a^{2}}{c} t\right)$.

\section{Case 2.}

$$
\begin{array}{r}
a_{4}=0, p_{1}=i p_{3}, \quad p_{2}=-i p_{3}, \quad p_{4}=i \sqrt{3} p_{3}, \quad r_{1}=i p_{3}\left(\frac{3 a^{2}-16 b c p_{3}^{4}}{c}\right), \\
r_{2}=i p_{3}\left(\frac{16 b c p_{3}^{4}-3 a^{2}}{c}\right), r_{3}=p_{3}\left(\frac{3 a^{2}-16 b c p_{3}^{4}}{c}\right), r_{4}=\frac{i \sqrt{3} p_{3}\left(3 a^{2}+16 b c p_{3}^{4}\right)}{c},
\end{array}
$$

where $a_{1}, a_{2}, a_{3}$ and $p_{3}$ are free parameters. Substituting (50) into Eq. (47), we can obtain

$q(x, t)=\frac{a_{1} p_{3}^{2} \cosh \left(\xi_{3}\right)-i a_{2} p_{3}^{2} \sinh \left(\xi_{3}\right)+a_{3} p_{3}^{2} \cosh \left(\xi_{3}\right)-3 p_{3}^{2} \exp \left(-\xi_{4}\right)}{a_{1} \cosh \left(\xi_{3}\right)-i a_{2} \sinh \left(\xi_{3}\right)+a_{3} \cosh \left(\xi_{3}\right)+\exp \left(-\xi_{4}\right)}+\left(\frac{a_{1} p_{3} \sinh \left(\xi_{3}\right)-i a_{2} p_{3} \cosh \left(\xi_{3}\right)+a_{3} p_{3} \sinh \left(\xi_{3}\right)-i \sqrt{3} \exp \left(-\xi_{4}\right)}{a_{1} \cosh \left(\xi_{3}\right)-i a_{2} \sinh \left(\xi_{3}\right)+a_{3} \cosh \left(\xi_{3}\right)+\exp \left(-\xi_{4}\right)}\right)^{2}$,

where $\xi_{3}=p_{3}\left(x-\frac{16 b c p_{3}^{4}-3 a^{2}}{c} t\right)$ and $\xi_{4}=i \sqrt{3} p_{3}\left(x+\frac{3 a^{2}+16 b c p_{3}^{4}}{c} t\right)$.

\section{Case 3.}

$$
p_{1}=p_{2}=i p_{3}, p_{3}=p_{4}, r_{1}=r_{2}=i p_{4}\left(\frac{3 a^{2}-16 b c p_{4}^{4}}{c}\right), r_{3}=r_{4}=p_{4}\left(\frac{3 a^{2}-16 b c p_{4}^{4}}{c}\right),
$$


where $a_{1}, a_{2}, a_{3}, a_{4}$ are free parameters. Substituting (52) into Eq. (47), we have the following solutions:

$$
q(x, t)=p_{4}^{2}\left(1-\frac{a_{1} \sinh \left(\xi_{4}\right)+i a_{2} \cosh \left(\xi_{4}\right)+a_{3} \sinh \left(\xi_{4}\right)-\exp \left(-\xi_{4}\right)+a_{4} \exp \left(\xi_{4}\right)}{a_{1} \cosh \left(\xi_{4}\right)+i a_{2} \sinh \left(\xi_{4}\right)+a_{3} \cosh \left(\xi_{4}\right)+\exp \left(-\xi_{4}\right)+a_{4} \exp \left(\xi_{4}\right)}\right)^{2}
$$

where $\xi_{3}=p_{3}\left(x-\frac{16 b c p_{4}^{4}-3 a^{2}}{c} t\right)$. Also, if $a_{1}=a_{3}=-1, a_{4}=1$, then Eq. (53) can be reduced to the following form:

$$
q(x, t)=-p_{4}^{2} \operatorname{cosech}^{2}\left(\xi_{4}\right)
$$

\section{Case 4.}

$$
a_{1}=a_{4}=0, p_{2}=p_{3}=0, r_{3}=r_{2}=0, r_{4}=p_{4}\left(\frac{3 a^{2}-b c p_{4}^{4}}{c}\right),
$$

where $a_{2}, a_{3}$ are free parameters. Substituting (55) into Eq. (47) yields the exact solution

$$
q(x, t)=p_{4}^{2}\left(\frac{a_{3} \exp \left(-\xi_{4}\right)}{\left(a_{3}+\exp \left(\xi_{4}\right)\right)^{2}}\right)
$$

where $\xi_{4}=p_{4}\left(x-\frac{b c p_{4}^{4}-3 a^{2}}{c} t\right)$. Also, if we take $a_{3}=1$, then Eq. (56) can be reduced to the following form:

$$
q(x, t)=\frac{p_{4}^{2}}{2\left(1+\cosh \left(\xi_{4}\right)\right)}
$$

\section{Case 5.}

$$
\begin{gathered}
a_{1}=a_{4}=0, \quad p_{2}=i p_{3}, \quad p_{4}=i \sqrt{3} p_{3}, \quad r_{1}=i p_{3}\left(\frac{3 a^{2}-16 b c p_{3}^{4}}{c}\right), \\
r_{3}=p_{3}\left(\frac{3 a^{2}-16 b c p_{3}^{4}}{c}\right), r_{4}=\frac{i \sqrt{3} p_{3}\left(3 a^{2}+16 b c p_{3}^{4}\right)}{c}
\end{gathered}
$$

where $a_{2}, a_{3}$ are free parameters. Substituting (58) into Eq. (47) yields the solution

$q(x, t)=\frac{i a_{2} p_{3}^{2} \sinh \left(\xi_{3}\right)+a_{3} p_{3}^{2} \cosh \left(\xi_{3}\right)-3 p_{3}^{2} \exp \left(-\xi_{4}\right)}{i a_{2} \sinh \left(\xi_{3}\right)+a_{3} \cosh \left(\xi_{3}\right)+\exp \left(-\xi_{4}\right)}-\left(\frac{i a_{2} p_{3} \cosh \left(\xi_{3}\right)+a_{3} p_{3} \sinh \left(\xi_{3}\right)-i \sqrt{3} \exp \left(-\xi_{4}\right)}{i a_{2} \sinh \left(\xi_{3}\right)+a_{3} \cosh \left(\xi_{3}\right)+\exp \left(-\xi_{4}\right)}\right)^{2}$

where $\xi_{3}=p_{3}\left(x+\frac{16 b c p_{3}^{4}-3 a^{2}}{c} t\right)$ and $\xi_{4}=i \sqrt{3} p_{3}\left(x+\frac{3 a^{2}+16 b c p_{3}^{4}}{c} t\right)$.

\section{Case 6.}

$$
\begin{gathered}
a_{2}=a_{4}=0, \quad p_{1}=i p_{3}, \quad p_{4}=i \sqrt{3} p_{3}, \quad r_{1}=i p_{3}\left(\frac{3 a^{2}-16 b c p_{3}^{4}}{c}\right) \\
r_{3}=p_{3}\left(\frac{3 a^{2}-16 b c p_{3}^{4}}{c}\right), r_{4}=\frac{i \sqrt{3} p_{3}\left(3 a^{2}+16 b c p_{3}^{4}\right)}{c}
\end{gathered}
$$

where $a_{1}, a_{3}$ are free parameters. Substituting (60) into Eq. (47), we obtain solitary solution

$$
q(x, t)=\frac{a_{1} p_{3}^{2} \cosh \left(\xi_{3}\right)+a_{3} p_{3}^{2} \cosh \left(\xi_{3}\right)-3 p_{3}^{2} \exp \left(-\xi_{4}\right)}{a_{1} \cosh \left(\xi_{3}\right)+a_{3} \cosh \left(\xi_{3}\right)+\exp \left(-\xi_{4}\right)}-\left(\frac{a_{1} p_{3} \sinh \left(\xi_{3}\right)+a_{3} p_{3} \sinh \left(\xi_{3}\right)-i \sqrt{3} p_{3} \exp \left(-\xi_{4}\right)}{a_{1} \cosh \left(\xi_{3}\right)+a_{3} \cosh \left(\xi_{3}\right)+\exp \left(-\xi_{4}\right)}\right)^{2}
$$


where $\xi_{3}=p_{3}\left(x+\frac{16 b c p_{3}^{4}-3 a^{2}}{c} t\right)$ and $\xi_{4}=i \sqrt{3} p_{3}\left(x+\frac{3 a^{2}+16 b c p_{3}^{4}}{c} t\right)$. Also, if we take $a_{1}=$ $a_{3}=1$, then Eq. (61) can be reduced to the following form:

$$
q(x, t)=\frac{2 p_{3}^{2} \cosh \left(\xi_{3}\right)-3 p_{3}^{2} \exp \left(-\xi_{4}\right)}{2 \cosh \left(\xi_{3}\right)+\exp \left(-\xi_{4}\right)}-\left(\frac{2 p_{3} \sinh \left(\xi_{3}\right)-i \sqrt{3} p_{3} \exp \left(-\xi_{4}\right)}{2 \cosh \left(\xi_{3}\right)+\exp \left(-\xi_{4}\right)}\right)^{2}
$$

\section{Case 7.}

$$
a_{3}=a_{4}=0, \quad p_{1}=p_{2}, \quad p_{4}=\sqrt{3} p_{2}, r_{1}=r_{2}=p_{2}\left(\frac{3 a^{2}-16 b c p_{2}^{4}}{c}\right), r_{4}=\frac{\sqrt{3} p_{2}\left(3 a^{2}+16 b c p_{3}^{4}\right)}{c},
$$

where $a_{1}, a_{2}$ are free parameters. Substituting (63) into Eq. (47) yields the periodic solitary solution

$q(x, t)=\frac{-a_{1} p_{2}^{2} \cos \left(\xi_{2}\right)-a_{2} p_{2}^{2} \sin \left(\xi_{2}\right)+3 p_{2}^{2} \exp \left(-\xi_{4}\right)}{a_{1} \cos \left(\xi_{2}\right)+a_{2} \sin \left(\xi_{2}\right)+\exp \left(-\xi_{4}\right)}-\left(\frac{a_{1} p_{2} \sin \left(\xi_{2}\right)+a_{2} p_{2} \cos \left(\xi_{2}\right)-\sqrt{3} p_{2} \exp \left(-\xi_{4}\right)}{a_{1} \cos \left(\xi_{3}\right)+a_{2} \sin \left(\xi_{2}\right)+\exp \left(-\xi_{4}\right)}\right)^{2}$

where $\xi_{2}=p_{2}\left(x+\frac{16 b c p_{3}^{4}-3 a^{2}}{c} t\right)$ and $\xi_{4}=\sqrt{3} p_{3}\left(x+\frac{3 a^{2}+16 b c p_{3}^{4}}{c} t\right)$.

\section{Case 8.}

$$
a_{1}=a_{2}=a_{4}=0, \quad p_{4}=i \sqrt{3} p_{3}, r_{3}=p_{3}\left(\frac{3 a^{2}-16 b c p_{2}^{4}}{c}\right), r_{4}=\frac{i \sqrt{3} p_{3}\left(3 a^{2}+16 b c p_{3}^{4}\right)}{c},
$$

where $a_{3}$ is a free parameter. Substituting (65) into Eq. (47), we find

$$
q(x, t)=\frac{a_{3} p_{3}^{2} \cosh \left(\xi_{3}\right)-3 p_{3}^{2} \exp \left(-\xi_{4}\right)}{a_{3} \cosh \left(\xi_{3}\right)+\exp \left(-\xi_{4}\right)}-\left(\frac{a_{3} p_{3} \sinh \left(\xi_{3}\right)-i \sqrt{3} p_{3} \exp \left(-\xi_{4}\right)}{a_{3} \cosh \left(\xi_{3}\right)+\exp \left(-\xi_{4}\right)}\right)^{2},
$$

where $\xi_{3}=p_{3}\left(x+\frac{16 b c p_{3}^{4}-3 a^{2}}{c} t\right)$ and $\xi_{4}=i \sqrt{3} p_{3}\left(x+\frac{3 a^{2}+16 b c p_{3}^{4}}{c} t\right)$.

\section{Case 9.}

$$
a_{1}=a_{3}=a_{4}=0, \quad p_{4}=\sqrt{3} p_{2}, r_{3}=p_{2}\left(\frac{3 a^{2}-16 b c p_{2}^{4}}{c}\right), r_{4}=\frac{\sqrt{3} p_{2}\left(3 a^{2}+16 b c p_{3}^{4}\right)}{c},
$$

where $a_{2}$ is a free parameter. Substituting (67) into Eq. (47) yields the periodic solitary solution

$$
q(x, t)=\frac{-a_{2} p_{2}^{2} \sin \left(\xi_{2}\right)+3 p_{3}^{2} \exp \left(-\xi_{4}\right)}{a_{2} \sin \left(\xi_{2}\right)+\exp \left(-\xi_{4}\right)}-\left(\frac{a_{2} p_{2} \cos \left(\xi_{2}\right)-\sqrt{3} p_{2} \exp \left(-\xi_{4}\right)}{a_{2} \sin \left(\xi_{2}\right)+\exp \left(-\xi_{4}\right)}\right)^{2}
$$

where $\xi_{2}=p_{2}\left(x+\frac{16 b c p_{3}^{4}-3 a^{2}}{c} t\right)$ and $\xi_{4}=\sqrt{3} p_{2}\left(x+\frac{3 a^{2}+16 b c p_{3}^{4}}{c} t\right)$.

\section{Case 10.}

$$
a_{1}=0, \quad p_{2}=i p_{4}, p_{3}=p_{4}, \quad r_{2}=i p_{4}\left(\frac{3 a^{2}-16 b c p_{4}^{4}}{c}\right), r_{3}=r_{4}=p_{4}\left(\frac{3 a^{2}-16 b c p_{4}^{4}}{c}\right),
$$

where $a_{2}, a_{3}, a_{4}$ are free parameters. Substituting (69) into Eq. (47) yields the exact solution

$$
q(x, t)=p_{4}^{2}-\left(\frac{i a_{2} p_{4} \cosh \left(\xi_{4}\right)+a_{3} p_{4} \sinh \left(\xi_{4}\right)-p_{4} \exp \left(-\xi_{4}\right)+a_{4} p_{4} \exp \left(\xi_{4}\right)}{i a_{2} \sinh \left(\xi_{4}\right)+a_{3} \cosh \left(\xi_{4}\right)+\exp \left(-\xi_{4}\right)+a_{4} \exp \left(\xi_{4}\right)}\right)^{2}
$$


where $\xi_{4}=p_{4}\left(x+\frac{16 b c p_{4}^{4}-3 a^{2}}{c} t\right)$. Also, if we take $a_{3}=-1$ and $a_{4}=1$,then Eq. (70) can be reduced to the solution

$$
q(x, t)=p_{4}^{2}\left(1-\left(\frac{\sinh \left(\xi_{4}\right)+i a_{2} \cosh \left(\xi_{4}\right)}{\cosh \left(\xi_{4}\right)+i a_{2} \sinh \left(\xi_{4}\right)}\right)^{2}\right)
$$

\section{Case 11.}

$$
a_{2}=0, \quad p_{1}=i p_{4}, p_{3}=p_{4}, \quad r_{1}=i p_{4}\left(\frac{3 a^{2}-16 b c p_{4}^{4}}{c}\right), r_{3}=r_{4}=p_{4}\left(\frac{3 a^{2}-16 b c p_{4}^{4}}{c}\right),
$$

where $a_{1}, a_{3}, a_{4}$ are free parameters. Substituting (72) into Eq. (47) yields the solitary solution

$$
q(x, t)=p_{4}^{2}-\left(\frac{a_{1} p_{4} \sinh \left(\xi_{4}\right)+a_{3} p_{4} \sinh \left(\xi_{4}\right)-p_{4} \exp \left(-\xi_{4}\right)+a_{4} p_{4} \exp \left(\xi_{4}\right)}{a_{1} \sinh \left(\xi_{4}\right)+a_{3} \cosh \left(\xi_{4}\right)+\exp \left(-\xi_{4}\right)+a_{4} \exp \left(\xi_{4}\right)}\right)^{2},
$$

where $\xi_{4}=p_{4}\left(x+\frac{16 b c p_{4}^{4}-3 a^{2}}{c} t\right)$. Also, if we take $a_{1}=-1$ and $a_{3}=a_{4}=1$,then Eq. (73) can be reduced to the solution

$$
q(x, t)=p_{4}^{2}\left(1-\tanh ^{2}\left(\xi_{4}\right)\right)=p_{4}^{2} \operatorname{sech}^{2}\left(\xi_{4}\right)
$$

\section{Case 12.}

$$
a_{3}=0, \quad p_{1}=p_{2}=-i p_{4}, \quad r_{1}=r_{2}=-i p_{4}\left(\frac{3 a^{2}-16 b c p_{4}^{4}}{c}\right), r_{3}=p_{4}\left(\frac{3 a^{2}-16 b c p_{4}^{4}}{c}\right),
$$

where $a_{1}, a_{2}, a_{4}$ are free parameters. Substituting (75) into Eq. (47), we have

$$
q(x, t)=p_{4}^{2}-\left(\frac{a_{1} p_{4} \sinh \left(\xi_{4}\right)-i a_{2} p_{4} \cosh \left(\xi_{4}\right)-p_{4} \exp \left(-\xi_{4}\right)+a_{4} p_{4} \exp \left(\xi_{4}\right)}{a_{1} \cosh \left(\xi_{4}\right)-i a_{2} \sinh \left(\xi_{4}\right)+\exp \left(-\xi_{4}\right)+a_{4} \exp \left(\xi_{4}\right)}\right)^{2}
$$

where $\xi_{4}=p_{4}\left(x+\frac{16 b c p_{4}^{4}-3 a^{2}}{c} t\right)$. Also, if we take $a_{1}=-1$ and $a_{4}=1$,then Eq. (76) can be reduced to the solution

$$
q(x, t)=p_{4}^{2}\left(1-\left(\frac{\sinh \left(\xi_{4}\right)-i a_{2} \cosh \left(\xi_{4}\right)}{\cosh \left(\xi_{4}\right)-i a_{2} \sinh \left(\xi_{4}\right)}\right)^{2}\right)
$$

\section{Case 13.}

$$
a_{1}=a_{2}=0, \quad p_{4}=p_{3}, \quad, r_{3}=r_{4}=p_{3}\left(\frac{3 a^{2}-16 b c p_{3}^{4}}{c}\right)
$$

where $a_{3}, a_{4}$ are free parameters. Substituting (78) into Eq. (47), we get

$$
q(x, t)=p_{3}^{2}-\left(\frac{a_{3} p_{3} \sinh \left(\xi_{3}\right)-p_{3} \exp \left(-\xi_{3}\right)+a_{4} p_{3} \exp \left(\xi_{3}\right)}{a_{3} \cosh \left(\xi_{3}\right)+\exp \left(-\xi_{3}\right)+a_{4} \exp \left(\xi_{3}\right)}\right)^{2}
$$

where $\xi_{3}=p_{3}\left(x+\frac{16 b c p_{3}^{4}-3 a^{2}}{c} t\right)$. Also, if we take $a_{3}=-1$ and $a_{4}=1$,then Eq. (79) can be reduced to 


$$
q(x, t)=p_{3}^{2}\left(1-\tanh ^{2}\left(\xi_{3}\right)\right)=p_{3}^{2} \operatorname{sech}^{2}\left(\xi_{3}\right)
$$

Case 14.

$$
a_{1}=a_{3}=0, \quad p_{4}=i p_{2}, r_{2}=p_{2}\left(\frac{3 a^{2}-16 b c p_{2}^{4}}{c}\right), \quad r_{4}=i p_{2}\left(\frac{3 a^{2}-16 b c p_{2}^{4}}{c}\right)
$$

where $a_{2}, a_{4}$ are free parameters. Substituting (81) into Eq. (47), we can obtain

$$
q(x, t)=-p_{2}^{2}-\left(\frac{a_{2} p_{2} \cos \left(\xi_{2}\right)-i p_{2} \exp \left(-i \xi_{2}\right)+i a_{4} p_{2} \exp \left(i \xi_{2}\right)}{a_{2} \sin \left(\xi_{2}\right)+\exp \left(-i \xi_{2}\right)+a_{4} \exp \left(i \xi_{2}\right)}\right)^{2}
$$

where $\xi_{2}=p_{2}\left(x+\frac{16 b c p_{2}^{4}-3 a^{2}}{c} t\right)$. Also, if we take $a_{4}=1$, then Eq. (82) can be reduced to the wave solution

$$
q(x, t)=-p_{2}^{2}\left(1+\frac{a_{2} \cos \left(\xi_{2}\right)-2 \sin \left(\xi_{2}\right)}{a_{2} \sin \left(\xi_{2}\right)+2 \cos \left(\xi_{2}\right)}\right)^{2}
$$

\section{Case 15.}

$$
a_{2}=a_{3}=0, \quad p_{4}=i p_{1}, r_{1}=p_{1}\left(\frac{3 a^{2}-16 b c p_{1}^{4}}{c}\right), \quad r_{4}=-i p_{1}\left(\frac{3 a^{2}-16 b c p_{1}^{4}}{c}\right)
$$

where $a_{1}, a_{4}$ are free parameters. Substituting (84) into Eq. (47) yields the following exact solution

$$
q(x, t)=-p_{1}^{2}-\left(\frac{-a_{1} p_{1} \sin \left(\xi_{1}\right)+i p_{1} \exp \left(i \xi_{1}\right)-i a_{4} p_{1} \exp \left(-i \xi_{1}\right)}{a_{1} \cos \left(\xi_{1}\right)+\exp \left(i \xi_{1}\right)+a_{4} \exp \left(-i \xi_{1}\right)}\right)^{2}
$$

where $\xi_{1}=p_{1}\left(x+\frac{16 b c p_{1}^{4}-3 a^{2}}{c} t\right)$. Also, if we take $a_{1}=-1$ and $a_{4}=1$, then we can reduce Eq. (85) to the following form:

$$
q(x, t)=-p_{1}^{2} \sec ^{2}\left(\xi_{1}\right)
$$

\section{Case 16.}

$$
a_{1}=a_{2}=a_{3}=0, \quad r_{4}=p_{4}\left(\frac{3 a^{2}-16 b c p_{4}^{4}}{c}\right)
$$

where $a_{4}$ is a free parameter. Substituting (87) into Eq. (47) yields soliton solution

$$
q(x, t)=p_{4}^{2}-\left(\frac{-p_{4} \exp \left(-\xi_{4}\right)+a_{4} p_{4} \exp \left(\xi_{4}\right)}{\exp \left(-\xi_{4}\right)+a_{4} \exp \left(\xi_{4}\right)}\right)^{2}
$$

where $\xi_{4}=p_{4}\left(x+\frac{16 b c p_{4}^{4}-3 a^{2}}{c} t\right)$. Also, if we take $a_{4}=1$, then Eq. (88) can be reduced to the following form:

$$
q(x, t)=p_{4}^{2} \operatorname{sech}^{2}\left(\xi_{4}\right)
$$



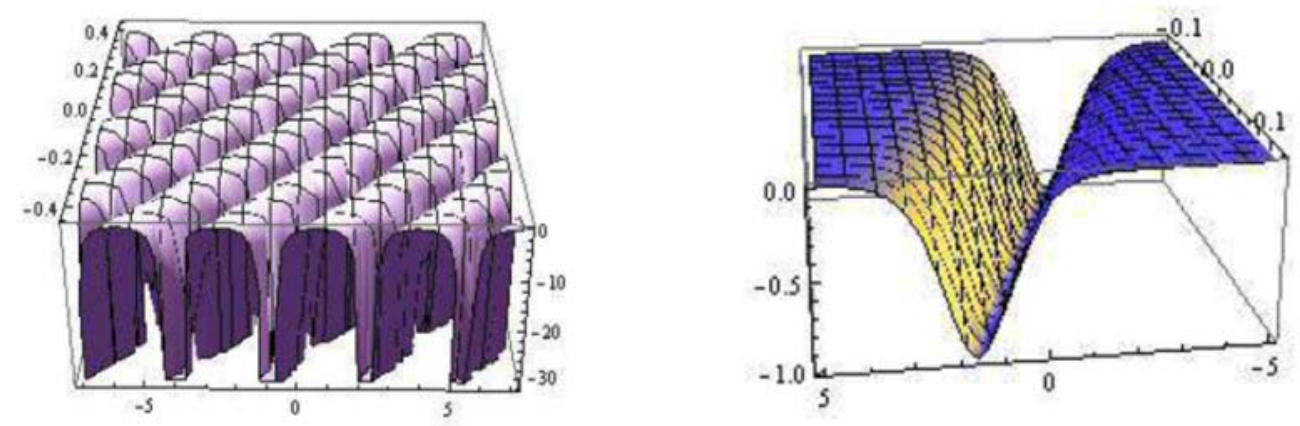

Figure 2. Periodic solution; Bright soliton

\section{Conclusion}

In this paper, we used the multi-wave method to obtain exact solutions to 5 th order $\mathrm{KdV}$ equation and fifth order $\mathrm{KdV}(\mathrm{fKdV})$ equation. Using a useful test function, we obtain new exact solitary solutions, such as M-type wave solution, periodic solitary wave solution, triangular periodic wave solution, etc., for two nonlinear evolution equations. So, these applications illustrate the effectiveness of the suggested method with the help of the Mathematica. The obtained results show that this approach is possible for integrable equations or non-integrable equations to have periodic solitary waves.

\section{References}

[1] Biswas, Anjan, Triki, Houria. "1-Soliton solution of the $\mathrm{D}(\mathrm{m} ; \mathrm{n})$ equation with generalized evolution." Applied Mathematics and Computation 217 (2011): 8482-8488.

[2] Hirota, Ryogo. "Exact solution of the KdV equation for multiple collisions of solitons." Physics Review Letters 27 (1971): 1192-1194.

[3] Hirota, Ryogo. "Exact N-soliton solution of the wave equation of long waves in shallow and nonlinear lattices." Journal of Mathematical Physics 14 (1973): 810-814.

[4] Gurefe, Yusuf, Misirli, Emine. "Exp-function method for solving nonlinear evolution equations with higher order nonlinearity." Computers and Mathematics with Applications 61 (2011): 2025-2030.

[5] Misirli, Emine, Gurefe, Yusuf. "Exp-function method for solving nonlinear evolution equations." Mathematical and Computational Applications 16 (2011): 258-266.

[6] Gurefe, Yusuf, Misirli, Emine. "New variable separation solutions of two-dimensional Burgers system.” Applied Mathematics and Computation 217 (2011): 9189-9197.

[7] Gurefe, Yusuf, Sonmezoglu, Abdullah, Misirli, Emine. "Application of the trial equation method for solving some nonlinear evolution equations arising in mathematical physics." Pramana-Journal of Physics 77 (2011): 1023-1029.

[8] Dai, Zhengde, Wang, Chuan-Jian, Lin, Song-Qing, Li, Dong-Long, Mu, Gui. "The three-wave method for nonlinear evolution equations." Nonlinear Science Letters A 1 (2010): 77-82.

[9] Shi, Yeqiong, Dai, Zhengde, Han Song, Huang Liwei. "The multi-wave method for nonlinear evolution equations." Mathematical and Computational Applications 15 (2010): 776-783. 
[10] Shi, Yeqiong, Li, Donglong. "New exact solutions for the $(2+1)$-dimensional Sawada Kotera equation." Computers and Fluids 68 (2012): 88-93.

[11] Gurefe, Yusuf, Pandir, Yusuf, Misirli, Emine. "New Exact Solutions of Stochastic KdV Equation." Applied Mathematical Sciences 6(65) (2012): 3225-3234.

[12] Girgis, Laila, Biswas Anjan. "Soliton perturbation theory for nonlinear wave equations." Applied Mathematics and Computation 216 (2010): 2226-2231.

[13] Biswas, Anjan, Konar, Swapan. "Soliton perturbation theory for the fifth order KdVtype equations with power law nonlinearity." Applied Mathematic Letters 20 (2007): 1122-1125. 